\title{
Secondary Central Nerve System Lymphoma With Intratumoral Hemorrhage Suggested as Intravascular Lymphoma by Autopsy: A Case Report
}

\author{
Yutaro Suzukia, Hiroaki Tanaka ${ }^{\text {b, e }}$, Kennichiro Suyama ${ }^{c}$, Hidetoshi Mochidac, \\ Yoshio Suzuki $^{\text {d }}$
}

\begin{abstract}
Intravascular large B-cell lymphoma (IVL) is a rare type of extranodal diffuse large B-cell lymphoma (DLBCL), which often infiltrates the central nervous system (CNS) during the clinical course. Cerebral hemorrhage in patients with CNS lymphoma at presentation is rare. Herein, we describe a case of secondary CNS lymphoma with intratumoral hemorrhage, which was suggested as IVL from autopsy findings. A 76-year-old Japanese man with a history of treatment for B-cell non-Hodgkin's lymphoma was transferred to our hospital in an ambulance for generalized convulsions. Brain CT scan revealed a high-density tumor with edema and intratumoral hemorrhage in the left temporal lobes. He died in a rapid course, and autopsy revealed a focal hemorrhage with diffuse infiltration of lymphoma cells in the left temporal lobe and findings suggestive of IVL. Furthermore, the autopsy revealed a discrepancy in the CD20 immunostaining of lymphoma cells between the brain and other organs. Clinicians should not eliminate CNS lymphoma from the differential diagnosis of intracranial tumor with hemorrhage. Although many patients with IVL have rapidly progressive courses, it is very important to diagnose IVL at the initial onset, even in serious situations, to consider CNS prophylaxis.
\end{abstract}

Keywords: Secondary central nervous system lymphoma; Intratumoral hemorrhage; Diffuse large B-cell lymphoma; Intravascular large B-cell lymphoma; Central nervous system prophylaxis; Discrepancy of CD20 immunostaining

\section{Introduction}

Intravascular large B-cell lymphoma (IVL) is a rare type of

\footnotetext{
Manuscript submitted August 26, 2017, accepted September 14, 2017

aDepartment of Internal Medicine, Asahi General Hospital, Chiba, Japan bepartment of Hematology, Asahi General Hospital, Chiba, Japan 'Department of Neurosurgery, Asahi General Hospital, Chiba, Japan dDepartment of Diagnostic Pathology, Asahi General Hospital, Chiba, Japan ${ }^{e}$ Corresponding Author: Hiroaki Tanaka, Department of Hematology, Asahi General Hospital, I-1326, Asahi-city, Chiba 289-2511, Japan.

Email: htanaka@hospital.asahi.chiba.jp
}

doi: https://doi.org/10.14740/jocmr3177w extranodal diffuse large B-cell lymphoma (DLBCL) characterized by the selective growth of lymphoma cells within the lumina of vessels, particularly capillaries [1]. It is a rapidly progressive, often fatal, and aggressive lymphoma that often infiltrates the central nervous system (CNS) during the clinical course. The reported risk of CNS recurrence at 3 years was $25 \%$ [2] and patients with any type of DLBCL have a $5 \%$ overall risk of CNS relapse [3].

CNS lymphoma consists of two types: primary CNS lymphoma (PCNSL) and secondary CNS lymphoma. Most CNS lymphomas are pathologically DLBCL. PCNSL represents all primary intracerebral or intraocular lymphomas at presentation and accounts for approximately $2-3 \%$ of all brain tumors [4]. Secondary CNS lymphoma is defined as CNS involvement by systemic lymphoma.

Here, we describe a secondary CNS lymphoma with intratumoral hemorrhage, which was suggested as intravascular lymphoma by autopsy.

\section{Case Report}

A 76-year-old Japanese man suffered from fever in April 2014. He had been receiving insulin therapy for diabetes mellitus and had been administered antiplatelet agent since the onset of cerebral infarction at age 60 . On physical examination, his superficial lymph nodes, liver, and spleen were not palpable. Laboratory findings were as follows: white blood cell (WBC) count, $8.5 \times 10^{9} / \mathrm{L}$ with $0.5 \%$ abnormal lymphocytes; hemoglobin, $7.8 \mathrm{~g} / \mathrm{dL}$; platelet count, $79 \times 10^{9} / \mathrm{L}$; lactate dehydrogenase (LDH), 1,004 U/L (normal range, 124 - $226 \mathrm{IU} / \mathrm{L}$ ); C-reactive protein (CRP), $19.02 \mathrm{mg} / \mathrm{dL}$; and soluble interleukin-2 receptor (sIL-2R) level, 11,400 IU/mL. Bone marrow aspiration revealed proliferation of large abnormal lymphocytes with vacuoles. These cells were positive for CD19, CD20, and kappa and were negative for CD5, CD10, and lambda, as determined by flow cytometric analysis. Positron emission tomographycomputed tomography (PET/CT) scans revealed abnormal accumulations in the spleen and bone marrow. He was diagnosed with B-cell non-Hodgkin's lymphoma. Because of acute deterioration, chemotherapy had to be initiated despite the absence of a pathological definitive diagnosis with bone marrow biopsy. Eight courses of reduced-dose R-CHOP therapy, which consisted of rituximab (375 mg/m² on day 1), doxorubicin (25 


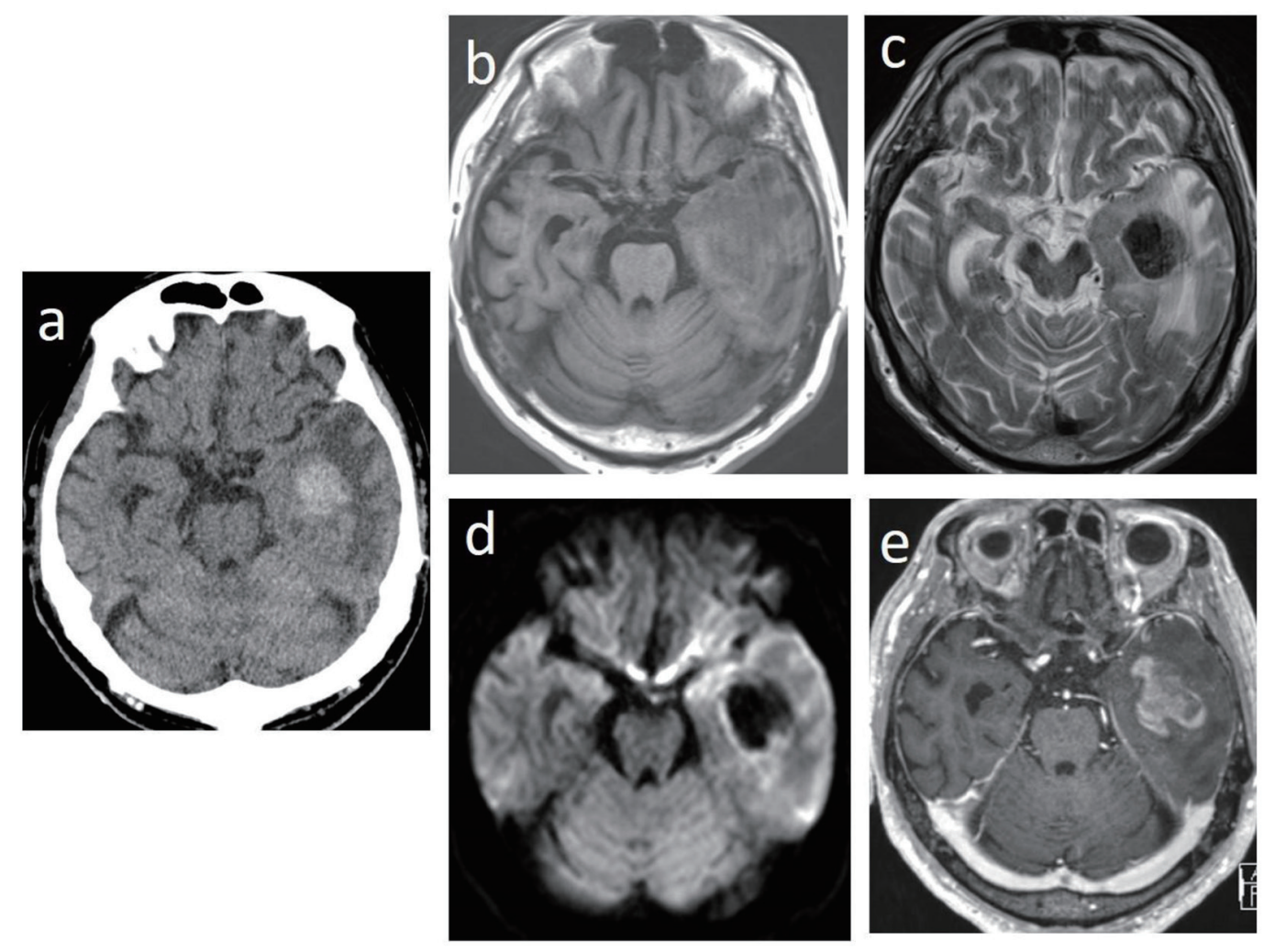

Figure 1. Brain computed tomographic images showing a high-density tumor with edema and intratumoral hemorrhage in the left temporal lobes (a). The tumor is iso- to hypointense on T1-weighted magnetic resonance imaging (MRI) (b) and hypointense on T2-weighted MRI (c) and on diffusion-weighted MRI (d). Gadolinium-enhanced T1-weighted MRI showing heterogeneous enhancement (e).

$\mathrm{mg} / \mathrm{m}^{2}$ on day 2$)$, cyclophosphamide $\left(375 \mathrm{mg} / \mathrm{m}^{2}\right.$ on day 2$)$, vincristine $\left(0.7 \mathrm{mg} / \mathrm{m}^{2}\right.$ on day 2$)$, and prednisone $(50 \mathrm{mg} / \mathrm{body}$ on days 1 - 5), were administered every 3 weeks. CNS prophylaxis was not performed. Complete remission was confirmed by laboratory findings and PET/CT. Until April 2015, there were no findings that actively suggested relapse. His sIL-2R level had remained approximately $700-900 \mathrm{IU} / \mathrm{mL}$ and was $876 \mathrm{IU} / \mathrm{mL}$ at that time.

In May 2015, 3 days before admission, he could not recognize cards in a deck when playing by himself. Additionally, behaviors that his family could not understand became increasingly evident. On the day of admission, he moaned in the bedroom and then had generalized convulsions. He was transferred to our hospital in an ambulance while the convulsion persisted. On physical examination at emergency visits, tonic-clonic convulsions continued. His temperature was $40.5^{\circ} \mathrm{C}$, heart rate was 157 beats per minute, blood pressure was $187 / 79 \mathrm{~mm} \mathrm{Hg}$, and respiratory rate was 20 breaths per minute. His Glasgow coma scale score was E1V1M4. His pupils were round and equal with a slow light reflex. There was right conjugate deviation but no neck stiffness. The liver, spleen, and superficial lymph nodes were not palpable. The laboratory findings were as follows: WBC count, $15.1 \times 10^{9} / \mathrm{L}$ with no abnormal cells; hemoglobin, $9.9 \mathrm{~g} / \mathrm{dL}$; platelet count, $188 \times 10^{9} / \mathrm{L}$; prothrombin time, $13.2 \mathrm{~s}$; activated partial thromboplastin time, $27.2 \mathrm{~s}$; fibrinogen, $417 \mathrm{mg} / \mathrm{dL}$; D-dimer, 2.5 $\mu \mathrm{g} / \mathrm{mL}$; total protein, $7.0 \mathrm{~g} / \mathrm{dL}$; albumin, $4.5 \mathrm{~g} / \mathrm{dL}$; aspartate aminotransferase, $24 \mathrm{U} / \mathrm{L}$; alanine aminotransferase, $11 \mathrm{U} / \mathrm{L}$; LDH, $313 \mathrm{U} / \mathrm{L}$; total bilirubin, $0.5 \mathrm{mg} / \mathrm{dL}$; serum creatinine, $1.36 \mathrm{mg} / \mathrm{dL}$; CRP, $0.75 \mathrm{mg} / \mathrm{dL}$; HbA1c, $8.3 \%$; and electrolytes and blood glucose levels were normal. Brain $\mathrm{CT}$ scan revealed a high-density tumor with edema and intratumoral hemorrhage in the left temporal lobes (Fig. 1a). The tumor was iso- to hypointense on T1-weighted magnetic resonance imaging (MRI) and hypointense on T2-weighted MRI and diffusion-weighted MRI (Fig. 1b-d). In addition, gadolinium-enhanced T1-weighted imaging showed heterogeneous enhancement (Fig. 1e).

For convulsion, diazepam and levetiracetam were initiated. Brain biopsy for definitive diagnosis was scheduled. However, convulsion frequently developed despite increased amounts of levetiracetam. His urinary tract infection was complicated, so antibiotics were started; however, his fever worsened. On the 24th hospital day, his LDH level drastically increased to 1,792 $\mathrm{U} / \mathrm{L}$ and his CRP increased to $23.5 \mathrm{mg} / \mathrm{dL}$. A few lymphoma cells were detected in the peripheral blood. Respiratory failure and disseminated intravascular coagulation (DIC) developed. He died on the 27th hospital day.

Autopsy was performed with the consent of his family and revealed DLBCL, which had infiltrated the brain, meninges, bone marrow, spleen, lung, retroperitoneum, adrenal gland, and lymph nodes. However, there was no macroscopically enlarged lymph node. Focal hemorrhage was observed in the 

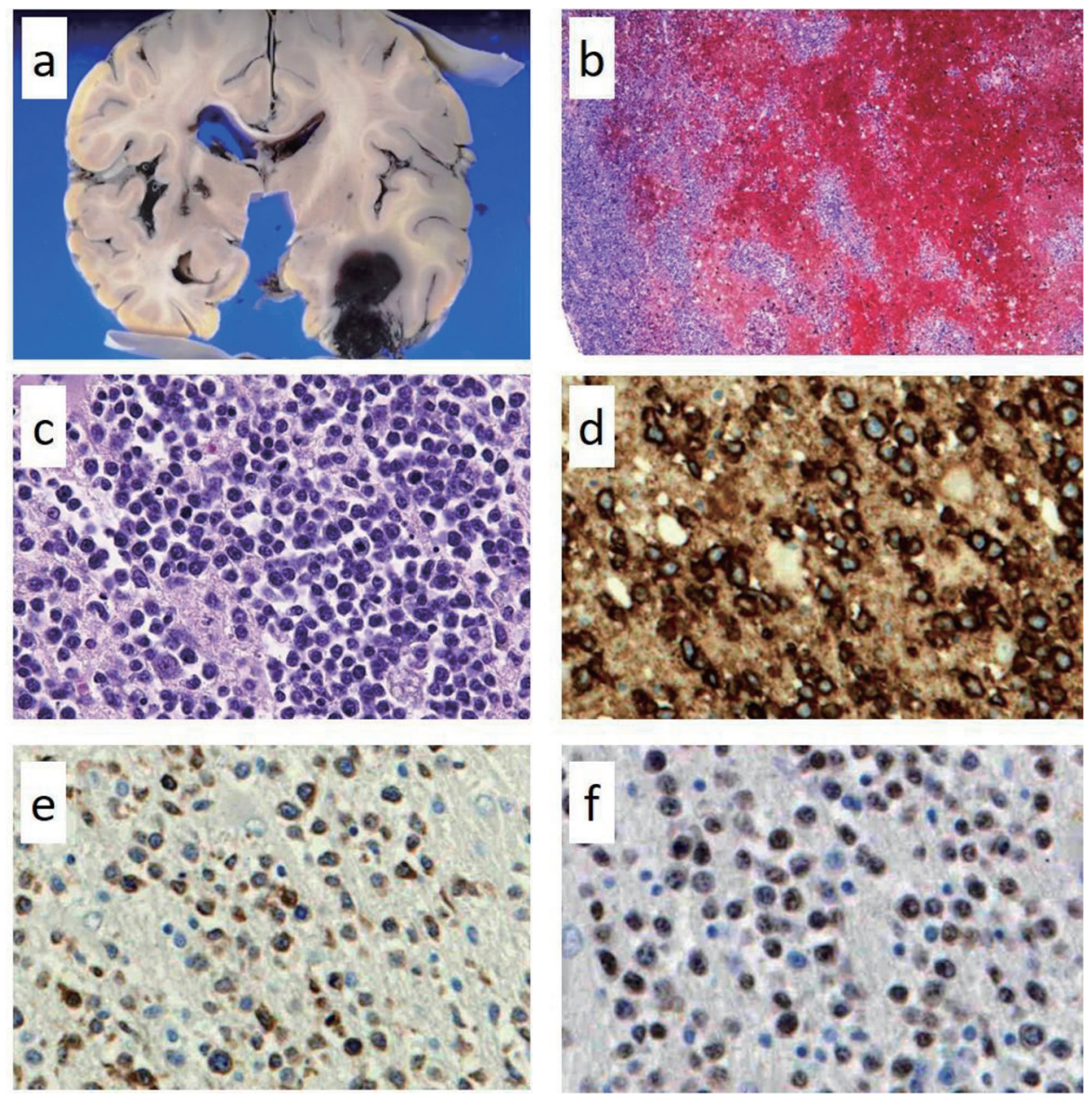

Figure 2. Pathological findings of autopsy in the left temporal lobe. Focal hemorrhages in the left temporal lobe (a) in which lymphoma cells had diffusely infiltrated with hemorrhage; (b) hematoxylin and eosin staining $\times 100$; (c) $\times 400$. The lymphoma cells in the brain lesion are positive for CD20 (d, × 400); CD79a (e, $\times 400)$; and PAX5 (f, $\times 400)$.

left temporal lobe in which lymphoma cells had diffusely infiltrated with hemorrhage (Fig. 2a-c). Lymphoma cells were observed in parts of the lung lesions within the lumina of vessels (Fig. 3a). Lymphoma cells in the brain lesion were positive for CD20, CD79a, and PAX5 (Fig. 2d-f), and those of other organs were negative for CD20 but positive for PAX5 (Fig. 3b, c).

\section{Discussion}

There were two notable points in our case. The first was that there were some findings suggestive of IVL. The autopsy revealed lymphoma cells within the lumina of vessels in sections of the lung lesions. It is unlikely that this finding was due to a leukemic change of the lymphoma because only $3 \%$ of the lymphoma cells were found in the peripheral blood immediately before death. The autopsy also revealed lymphoma cell invasion into the bone marrow, peripheral blood, spleen, adrenal grand, lung, and brain without macroscopically enlarged lymph nodes. Lymphoma cells of IVL often infiltrate these organs [1]. IVL complicates CNS mass lesions during their clinical course, and the overall features of these IVL lesions are very similar to or indistinguishable from those of PCNSL [5].
Although our patient could not be pathologically diagnosed with IVL based on the autopsy findings alone, it is clinically probable that his lymphoma would have been IVL since the first onset because LDH and sIL-2R value were remarkably increased and there were no enlarged lymph nodes at that time. Although many patients with IVL have rapidly progressive courses, it is very important to diagnose IVL at the initial onset, even in serious situations, to consider CNS prophylaxis. IVL involves bone marrow in $74 \%$ of patients with Asian-variant IVL and $30 \%$ of patients with Western-variant IVL [6]. The efficacy of skin biopsy in the diagnosis of IVL has been reported [7]. Because biopsy of these organs can be performed in patients with poor general status, even if a patient has a poor general status, at least bone marrow and skin biopsy should be performed for the early diagnosis of IVL.

The autopsy revealed a discrepancy in the CD20 immunostaining of lymphoma cells between the brain and other organs. A CD20-negative phenotypic change has been previously observed after chemotherapies with rituximab in a number of patients with CD20-positive B-cell lymphoma [8]. Here, the lymphoma cells in the organs other than the brain were exposed to rituximab therapy and may have recurred following downregulation of CD20 expression. In addition, the 


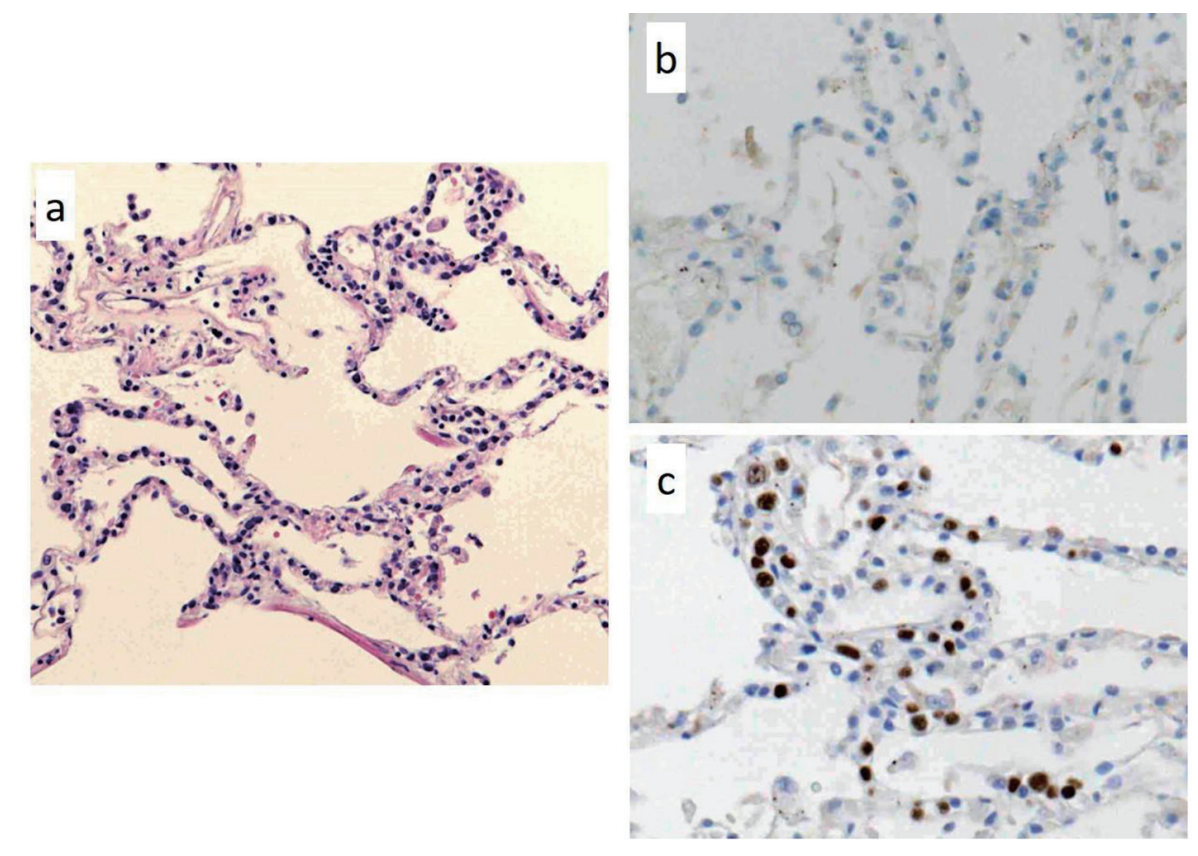

Figure 3. Pathological findings of autopsy in a part of the lung lesions. There are lymphoma cells within the lumina of vessels; (a) hematoxylin and eosin staining ( $\times 100)$. The lymphoma cells in the lung lesion are negative for CD20 (b, $\times 400)$, but positive for PAX5 (c, × 400).

lymphoma cells in the brain may have infiltrated into the cerebrospinal fluid at first presentation and not have been exposed to rituximab because of the blood-brain barrier. It is interesting that these two different clones of lymphoma cells recurred at approximately the same time. Another hypothesis involves the possible masking of CD20 staining by rituximab. Jilani et al reported that rituximab therapy leads to a substantial but transient downregulation of CD20 expression by demonstrating that CD20 expression in cultured cells restores within $24 \mathrm{~h}$ of exposure to rituximab [9]. In our case, however, rituximab therapy had already been completed 6 months before the hemorrhage.

The secondary notable point in our case was the cerebral hemorrhage in a patient with CNS lymphoma that presented with atypical imaging findings. Cerebral hemorrhage due to brain tumor is not uncommon in patients with metastatic brain tumor and glioblastoma. However, cerebral hemorrhage in patients with CNS lymphoma at presentation is quite rare, with only a few published case reports [10-14]. Several hypotheses have been proposed to explain the causes of cerebral hemorrhage in patients with CNS lymphoma. Some reports of PCNSL with intratumoral hemorrhage have suggested that a high level of vascular endothelial growth factor immunoreactivity in lymphoma cells is correlated with intratumoral hemorrhage $[10,15]$. Additionally, some case reports of IVL with cerebral hemorrhage have described possible causes of cerebral hemorrhage, such as a complication of DIC [16], obstruction of the lumen of the vessel due to complete fullness of lymphoma cells [17], and an injury of the vessel wall due to direct interaction between lymphoma cells and endothelial cells of the cerebral vessel [12]. Streletz et al stated that when stroke and hemorrhage occur with a CNS lymphoma, it is usually IVL
[10].

Generally, CNS lymphoma lesions are typically hypo- or isointense on T1-weighted MRI, and iso- to hyperintense on T2-weighted MRI, but they are often hypointense in gray matter. Most lesions show moderate-to-marked contrast enhancement on CT and MRI $[18,19]$. On diffusion-weighted MRI, the lesions are hyperintense because CNS lymphomas are highly cellular tumors and water diffusion is often restricted [20]. In our case, because of a decrease in the cell density, which was caused by intratumoral hemorrhage, the CNS lymphoma lesions were hypointense on diffusion-weighted imaging. CNS lymphomas with intratumoral hemorrhage are rare; however, clinicians should not eliminate CNS lymphoma from the differential diagnosis of intracranial tumors with hemorrhage. In our case, there had been long-term administration of an antiplatelet agent and a past medical history of cerebral infarction and long-term diabetes mellitus. These factors, other than the CNS lymphoma itself, may have influenced his cerebral hemorrhage.

In conclusion, we encountered a case of secondary CNS lymphoma with intratumoral hemorrhage, which was suggested as IVL on the basis of the autopsy findings. It is very important to diagnose IVL at the first onset, even in serious situations, to consider CNS prophylaxis. CNS lymphomas with intratumoral hemorrhage are rare; however, clinicians should not eliminate CNS lymphoma from the differential diagnosis of intracranial tumors with hemorrhage.

\section{Conflict of Interest}

All authors declare no conflict of interest. 


\section{References}

1. Nakamura S, Ponzoni M, Campo E. Intravascular large B-cell lymphoma. In: Swerdlow SH, Campo E, Harris NL, Jaffe ES, Pileri SA, Stein H, Thiele J, Vardiman JW, editors. WHO classification of tumours of haematopoietic and lymphoid tissues. 4th ed. Lyon: IARC; 2008. p. 252253.

2. Shimada K, Murase T, Matsue K, Okamoto M, Ichikawa N, Tsukamoto N, Niitsu N, et al. Central nervous system involvement in intravascular large B-cell lymphoma: a retrospective analysis of 109 patients. Cancer Sci. 2010;101(6):1480-1486.

3. Hill QA, Owen RG. CNS prophylaxis in lymphoma: who to target and what therapy to use. Blood Rev. 2006;20(6):319-332.

4. Kluin PM, Deckert M, Feery JM. Primary diffuse large B-cell lymphoma of the CNS In: Swerdlow SH, Campo E, Harris NL, Jaffe ES, Pileri SA, Stein H, Thiele J, et al. editors. WHO classification of tumours of haematopoietic and lymphoid tissues. 4th ed. Lyon: IARC; 2008. p. 240241.

5. Imai H, Kajimoto K, Taniwaki M, Miura I, Hatta $\mathrm{Y}$, Hashizume Y, Watanabe M, et al. Intravascular large Bcell lymphoma presenting with mass lesions in the central nervous system: a report of five cases. Pathol Int. 2004;54(4):231-236.

6. Ferreri AJ, Dognini GP, Campo E, Willemze R, Seymour JF, Bairey O, Martelli M, et al. Variations in clinical presentation, frequency of hemophagocytosis and clinical behavior of intravascular lymphoma diagnosed in different geographical regions. Haematologica. 2007;92(4):486492.

7. Matsue K, Asada N, Odawara J, Aoki T, Kimura S, Iwama K, Fujiwara H, et al. Random skin biopsy and bone marrow biopsy for diagnosis of intravascular large B cell lymphoma. Ann Hematol. 2011;90(4):417-421.

8. Hiraga J, Tomita A, Sugimoto T, Shimada K, Ito M, Nakamura S, Kiyoi H, et al. Down-regulation of CD20 expression in B-cell lymphoma cells after treatment with rituximab-containing combination chemotherapies: its prevalence and clinical significance. Blood. 2009;113(20):4885-4893.

9. Jilani I, O'Brien S, Manshuri T, Thomas DA, Thomazy
VA, Imam M, Naeem S, et al. Transient down-modulation of CD20 by rituximab in patients with chronic lymphocytic leukemia. Blood. 2003;102(10):3514-3520.

10. Streletz LJ, Terzic D, Salem K, Raza A, Deleu DT. CNS lymphoma masquerading as hemorrhagic stroke. Clin Neurol Neurosurg. 2012;114(3):262-264.

11. Rubenstein J, Fischbein N, Aldape K, Burton E, Shuman $M$. Hemorrhage and VEGF expression in a case of primary CNS lymphoma. J Neurooncol. 2002;58(1):53-56.

12. Passarin MG, Wen PY, Vattemi E, Buffone E, Ghimenton $\mathrm{C}$, Bontempini L, Ottaviani S, et al. Intravascular lymphomatosis and intracerebral haemorrhage. Neurol Sci. 2010;31(6):793-797.

13. Kimura N, Ishibashi M, Masuda T, Morishige M, Abe T, Fujiki M, Kashima K, et al. Primary central nervous system lymphoma with cortical laminar hemorrhage. J Neurol Sci. 2009;287(1-2):281-284.

14. Kim IY, Jung S, Jung TY, Kang SS, Choi C. Primary central nervous system lymphoma presenting as an acute massive intracerebral hemorrhage: case report with immunohistochemical study. Surg Neurol. 2008;70(3):308311.

15. Matsuyama J, Ichikawa M, Oikawa T, Sato T, Kishida Y, Oda K, Maeda T, et al. Primary CNS lymphoma arising in the region of the optic nerve presenting as loss of vision: 2 case reports, including a patient with a massive intracerebral hemorrhage. Brain Tumor Pathol. 2014;31(3):222228.

16. Lui PC, Wong GK, Poon WS, Tse GM. Intravascular lymphomatosis. J Clin Pathol. 2003;56(6):468-470.

17. Haninger DM, Davis TA, Parker JR, Slone SP, Parker JC, Jr. Intravascular large B-cell lymphoma presenting as acute hemorrhagic cerebral infarct with delirium. Ann Clin Lab Sci. 2013;43(3):305-310.

18. Zhang D, Hu LB, Henning TD, Ravarani EM, Zou LG, Feng XY, Wang WX, et al. MRI findings of primary CNS lymphoma in 26 immunocompetent patients. Korean $\mathbf{J}$ Radiol. 2010;11(3):269-277.

19. Go JL, Lee SC, Kim PE. Imaging of primary central nervous system lymphoma. Neurosurg Focus. 2006;21(5):E4.

20. Haldorsen IS, Espeland A, Larsson EM. Central nervous system lymphoma: characteristic findings on traditional and advanced imaging. AJNR Am J Neuroradiol. 2011;32(6):984-992. 